\title{
Seleção para resistência de genótipos de cenoura aos nematóides-das-galhas
}

\author{
Giovani O da Silva ${ }^{1}$; Jadir B Pinheiro²; Jairo V Vieira²; Agnaldo DF de Carvalho²
}

${ }^{1}$ Embrapa Hortaliças-SNT, C. Postal 317, 89460-000 Canoinhas-SC; olegario@cnph.embrapa.br (autor correspondente); ${ }^{2}$ Embrapa Hortaliças, C. Postal 218, 70359-970 Brasília-DF; jadir@cnph.embrapa.br; jairo@cnph.embrapa.br; agnaldo@cnph.embrapa.br

\section{RESUMO}

Foi verificada a relação entre caracteres avaliados em campo e casa-de-vegetação, relativos à avaliação de genótipos de cenoura quanto à resistência ao nematóide-das-galhas (Meloidogyne spp.), visando identificar quais caracteres possibilitam a diferenciação entre os genótipos e verificar a possibilidade de avaliação apenas em um ambiente ou a eliminação de caracteres que apresentem correlação alta. Avaliaram-se 38 progênies da população '0812518' e 31 progênies da população '0812519', além das cultivares Brasília e Kuronan como testemunhas tolerante e suscetível, respectivamente. Em campo naturalmente infestado por uma mistura de Meloidogyne incognita raça 1 e Meloidoigyne javanica, a seleção para resistência ao nematóide-das-galhas foi realizada com base na menor porcentagem de infecção na raiz principal dos genótipos de cenoura, rendimento de raiz e o fator de reprodução, enquanto que em casa-de-vegetação, para cada uma das mesmas espécies de nematóides e também para a mistura de ambas, foi medido o índice de galhas e índice de massa de ovos. Verificou-se que não houve possibilidade de seleção em apenas um dos ambientes testados ou a eliminação de caracteres, indicando que para a obtenção de cultivares mais produtivas e mais tolerantes é necessária a seleção combinada nos diferentes caracteres e ambientes. Para o experimento realizado em campo foi possível diferenciar os genótipos apenas para o rendimento de raiz. No experimento em casa-de-vegetação, para todos os caracteres foi possível identificar genótipos superiores. Porém, as médias das populações não foram melhores que a testemunha 'Brasília' da qual estas se originaram, confirmando a necessidade da busca por métodos mais eficientes de seleção.

Palavras-chave: Daucus carota, Meloidogyne incognita, Meloidoigyne javanica, fator de reprodução.

\begin{abstract}
Selection for carrot genotypes resistance to root-knot nematodes in field and greenhouse
\end{abstract}

We verified the relation among the evaluated characters in field and greenhouse, concerning to the evaluation to root-knot nematode (Meloidogyne spp.) for the carrot crop, to identify for which ones it is possible to differentiate among genotypes and to verify the possibility of evaluation only in one environment or the elimination of characters with high correlation. Thirty eight and thirty one families from the carrot populations '0812518' and '0812519', and the cultivars Brasília and Kuronan as tolerant and susceptible standards, respectively, were evaluated. In a field whose soil was naturally infected by a mix of Meloidogyne incognita race 1 and Meloidogyne javanica, the selection to resistance to root-knot nematode was based on lower percentage of infection in the root, of the major genotypes of carrot production in $\mathrm{t} / \mathrm{ha}$ and reproduction factor; and in greenhouse, to each one of the same nematode species and too for a mix of both, was evaluated the index of gall and index of egg mass. There is no possibility of selection in only one condition or the elimination of some characters, indicating that, to obtain more yielding and more tolerant cultivars, the combined selection in the various characters and environments are necessary. In the field experiment, it was only possible to differentiate the genotypes for root yield. In the greenhouse, it was possible to identify superior genotypes for all characters tested. However, the average population was not better than the 'Brasilia' standard that originated these populations. This result confirms the necessity to search for more efficient methods of selection.

Keywords: Daucus carota, Meloidogyne incognita, Meloidoigyne javanica, reproduction factor.

\section{(Recebido para publicação em 29 de abril de 2010; aceito em 18 de julho de 2011)}

(Received on April 29, 2010; accepted on July 18, 2011)

$\mathrm{O}$ s nematóides se constituem em um grave problema para o cultivo da cenoura em praticamente todas as regiões do mundo, com perdas que podem chegar a $100 \%$, dependendo de vários fatores tais como a densidade populacional, a suscetibilidade da cultivar, a espécie de nematóide, o tipo de solo e as condições ambientais. Todavia, mesmo em baixas infestações a produção pode ser comprometida em até $25 \%$. As perdas geralmente ocorrem devido à redução na quantidade e na qualidade do produto colhido. Em certas circunstâncias, a perda de peso nas raízes não é tão significativa quanto as deformações no formato, visto que esta parte da planta sofre alterações físicas e químicas em resposta ao ataque dos nematóides, com interferência direta na classificação comercial do produto (Huang et al., 1986a).

No Brasil, os danos mais significativos são resultantes do ataque dos nematóides-das-galhas, geralmente $M$. incognita e $M$. javanica que possuem maior distribuição territorial nas diferentes regiões brasileiras, embora outras espécies como M. hapla e M. arenaria sejam observadas em áreas isoladas do país.

Para o controle dos nematóide-das-galhas são utilizados métodos como a rotação de culturas, a aplicação de nematicidas e o emprego da resistência genética. Os nematicidas registrados no 
Brasil para a cenoura são granulados do grupo carbofuran, químico altamente tóxico e poluente do ambiente, inclusive da água do subsolo, além de aumentarem o custo de produção. O cultivo de leguminosas, crucíferas ou gramíneas não hospedeiras dos nematóides de galhas, previamente ao de cenoura, tem sido utilizado como medida de controle (Bruno et al, 2007; Charchar \& Aragão, 2003), porém não é eficaz em todas as situações, além de tornar o manejo mais complexo. A associação de mais de uma medida de controle na maioria das vezes é a melhor opção. A cultivar Brasília é conhecida mundialmente pela resistência genética que possui (Charchar et al., 2007a; Charchar et al., 2007b; Charchar et al., 2009).

A Embrapa Hortaliças lançou a cultivar 'BRS Brasília' em 1981, e posteriormente outras cultivares como 'BRS Alvorada', 'BRS Esplanada' e 'BRS Planalto' que possuem como uma das principais características a elevada resistência a Meloidogyne spp. observada em campo pelo lento desenvolvimento de galhas (Vieira et al., 2003; Vieira et al., 2005). Estudos de herança usando germoplasma derivado de 'BRS Brasília' indicaram que a resistência a $M$. javanica está condicionada por um gene dominante ou dois genes dominantes fortemente ligados (Simon et al., 2000).

A Embrapa tem buscado aprimorar as metodologias de avaliação de genótipos de cenoura para reação ao nematóides-das-galhas (Meloidogyne spp.). A seleção em campo, que é o método utilizado na instituição, possui a vantagem de possibilitar a seleção para outras características agronômicas; porém, pode conduzir a resultados insatisfatórios, já que estão sujeitos ao efeito da ausência de uma uniformidade de distribuição natural do inóculo. Além disto, é difícil a seleção para cada espécie de nematóide-das-galhas isoladamente, pois geralmente ocorre a mistura de espécies no campo e as populações de nematóides são bastante influenciadas pelo ambiente. Já a seleção em casa-de-vegetação possibilita o contorno destas desvantagens, porém tem custo alto, não possibilita a seleção para outras características agronômicas, além de ser mais trabalhosa e não refletir as condições de cultivo em campo.

O objetivo deste trabalho foi verificar a relação entre caracteres avaliados em campo e casa-de-vegetação, relativos à avaliação de genótipos de cenoura quanto à resistência ao nematóide-das-galhas (Meloidogyne spp.), identificar quais caracteres possibilitam a diferenciação entre os genótipos e verificar a possibilidade de avaliação apenas em um ambiente ou a eliminação de caracteres que apresentem correlação alta.

\section{MATERIAL E MÉTODOS}

Genótipos avaliados - $\mathrm{O}$ experimento foi realizado de novembro de 2008 a março de 2009, em campo experimental e em casa-de-vegetação, da Embrapa Hortaliças, Brasília-DF. Foram avaliadas as populações de cenoura ‘0812518' e ‘0812519' com 38 e 31 progênies respectivamente, as quais foram obtidas pela seleção em campo, para a mistura de populações de nematoides (M. incognita raça 1 e $M$. javanica), com a mesma metodologia descrita neste trabalho, por três anos (gerações) consecutivos. Ambas as populações são derivadas da cultivar Brasília. No total, avaliaram-se 71 genótipos (69 progênies e as testemunhas 'Brasilia', tolerante e 'Kuronan', suscetível) para resistência à população mista dos nematóide-das-galhas ( $M$. incognita raça 1 e $M$. javanica) em campo e casa-de-vegetação e para as duas espécies inoculadas isoladamente em casa-de-vegetação.

Experimento em campo - $\mathrm{O}$ experimento foi instalado em área de campo (Latossolo Vermelho Eutrófico) previamente infestada pela mistura populacional de $M$. incognita raça 1 e $M$. javanica. Para a inoculação do campo foi realizado o plantio do tomateiro silvestre suscetível (Solanum hirsutum) inoculado na mesma proporção com ambas as espécies, 120 dias antes da semeadura da cenoura. As plantas de tomate foram retiradas do local uma semana antes da implantação do experimento. $\mathrm{O}$ delineamento experimental utilizado foi de blocos casualizados com quatro repetições, e parcelas de $1 \mathrm{~m}^{2}$ com 100 plantas de cenoura.

A seleção das populações de ce- noura para resistência ao nematóide-das-galhas foi realizada 102 dias após a semeadura com base nas seguintes variáveis: menor porcentagem de infecção $(\% \mathrm{INF}=$ presença de galhas dos nematóides na raiz principal das plantas de progênies e cultivares/produção total de raízes*100); rendimento em $\mathrm{t} /$ ha e o fator de reprodução (FR). Para avaliação do fator de reprodução (FR), amostras simples de quatro pontos equidistantes de cada parcela foram coletadas inicialmente (antes da semeadura) e durante a colheita do experimento. Em seguida, as amostras foram reunidas para formar uma amostra composta de aproximadamente $0,5 \mathrm{~kg} /$ parcela. Estas amostras foram levadas para o laboratório da Embrapa Hortaliças e processadas de acordo com a técnica de Jenkins (1964), para extração dos juvenis de $2^{\circ}$ estádio de $M$. incognita raça 1 e $M$. javanica presentes no solo. O FR foi obtido pelo quociente entre a população final no solo coletada durante a colheita e a população inicial no solo antes da semeadura.

Experimento em casa-de-vegetação - Fêmeas dos nematóides-das-galhas pertencentes às espécies $M$. incognita raça 1 e $M$. javanica coletadas de raízes de tomateiro na área experimental da Embrapa Hortaliças e mantidos em casa-de-vegetação, foram submetidas a cortes perineais e os padrões descritos por Eisenback \& Hirschmann-Triantaphyllou (1991) foram tomados como referência para a identificação das espécies. Para análise do padrão da isoenzima esterase foi adaptada técnica com base no protocolo de Carneiro \& Almeida (2001).

Para a produção de inóculo, os nematóides foram multiplicados em plantas de tomate cv. 'Rutgers'. Dez dias após a germinação das sementes nas bandejas de isopor, foi realizado o transplante para vasos com capacidade para 3 L contendo substrato Plantmax ${ }^{\circledR}$ esterilizado. Três dias após o transplante foi realizada a inoculação das raízes das plântulas, com suspensão de 6.000 ovos e juvenis de segundo estádio (J2) de cada espécie isoladamente, em $5 \mathrm{~mL}$ de água distribuídos em volta do colo da planta.

Aos 45-50 dias após a inoculação, ovos e J2 das duas espécies de nematói- 
des foram extraídos dos sistemas radiculares das plantas de tomate cv. 'Rutgers' segundo metodologia de Bonetti \& Ferraz (1981). Este procedimento foi repetido duas vezes para máxima obtenção de ovos. A suspensão de ovos e juvenis foi recolhida com pipeta para um béquer e a contagem e calibração do inóculo foi feita em câmara de contagem, ao microscópio ótico.

Para a instalação do experimento realizou-se a semeadura das progênies de cenoura em copos plásticos com capacidade para $300 \mathrm{~mL}$. Quatorze dias após a semeadura, foi realizado o desbaste deixando apenas uma planta por copo. A inoculação foi realizada de acordo com o realizado para manutenção das espécies em plantas de tomateiro cv. 'Rutgers'. O experimento foi conduzido em delineamento inteiramente casualizado em esquema fatorial 69x3 (69 progênies de cenoura, duas espécies de nematóide e uma mistura populacional com a mesma proporção de cada espécie), com quatro repetições, sendo a unidade experimental uma planta por copo.

A avaliação das plantas foi realizada 48 dias após a inoculação. Foram avaliados: Índice de massa de ovos (IMO)= os sistemas radiculares lavados em água corrente foram coloridos por imersão em solução de Floxina B na proporção de 0,5 gramas/L de água, durante 15 minutos. Em seguida, foi realizada a contagem do número de massa de ovos dos nematóides sob microscópio estereoscópio no sistema radicular de cada planta/repetição (Dickson \& Struble, 1965). O IMO nas raízes foi obtido de acordo com Huang et al. (1986b), utilizando-se a escala de 1 a 5 ( $1=$ raízes sem massa de ovos; $2=$ raízes com 1 a 5 massas de ovos; $3=$ raízes com 6 a 15 massas de ovos; $4=$ raízes com 16 a 30 massas de ovos; e $5=$ raízes com mais de 30 massas de ovos); Índice de galhas $(\mathrm{IG})=$ o número de galhas em cada sistema radicular de cada planta/repetição foi quantificado. O IG nas raízes foi representado pela escala de 1 a 5 , de acordo com Charchar et al. (2003) (1= raiz sem galhas; $2=$ raiz com até 10 galhas pequenas; $3=$ raiz com até 50 galhas pequenas; $4=$ raiz com mais de 50 galhas pequenas e até 10 galhas grandes; e $5=$ raiz com mais de 50 galhas pequenas e mais de 10 galhas grandes). Galhas com mais de $3 \mathrm{~mm}$ foram consideradas grandes; Fator de reprodução (FR)= obtido pela divisão entre as densidades populacionais finais e iniciais $(\mathrm{FR}=\mathrm{Pf} /$ Pi) (Oostenbrink, 1966). Foi considerado como população inicial (Pi) o inóculo extraído, quantificado e calibrado para conter 6.000 ovos e juvenis por vaso.

Os dados obtidos no experimento de campo e de casa-de-vegetação foram submetidos à análise de homogeneidade de variância (teste de Bartllet), e de normalidade (Lilliefors), e transformados por arco seno da raiz quadrada da proporção, para atender a pressuposição de normalidade de distribuição. Para o experimento em casa-de-vegetação efetuou-se uma análise de variância para cada espécie separadamente e para a mistura populacional de $M$. incognita raça 1 e $M$. javanica de nematóides bem como a análise conjunta dos resultados, enquanto que para o experimento em campo, foi realizada análise de variância para a mistura de nematóides. Foi estimada ainda a correlação fenotípica entre todos os caracteres medidos em campo e em casa-de-vegetação, e agrupamento de médias dos tratamentos por Scott-Knott (1974), utilizando-se o programa Genes (Cruz, 2006).

\section{RESULTADOS E DISCUSSÃO}

Não houve diferença significativa para a variável fator de reprodução (FR) em campo. Para os demais caracteres avaliados no campo e em casa-de-vegetação houve diferenças significativas $(p<0,05)$ entre as progênies e/ ou testemunhas avaliadas (dados não mostrados).

O FR teve ainda elevado coeficiente de variação ambiental (28,98\%) com indicação de que essa variável teve seus resultados influenciados predominantemente por efeitos de ambiente. Em experimento realizado em campo por Covolo \& Benetti (1981), para avaliar a reação das cultivares de cenoura Nantes, Kuroda, Chantenay, Flaker e Danvers ao nematóide $M$. javanica, foi avaliado o número e peso de galhas, e observou-se que todas as cultivares foram suscetíveis a esta espécie. Segundo os autores, se o método de avaliação utilizado fosse mais preciso, com menor coeficiente de variação $(38,78 \%)$, poderia haver diferenças significativas.

No presente trabalho, porcentagem de infecção também demonstrou ser um caráter de forte influência ambiental $(21,11 \%)$, o que traz dificuldades na seleção, dado também os reduzidos valores de herdabilidade para estes dois caracteres. Para os demais caracteres os coeficientes de variação foram reduzidos (Tabela 1).

Na seleção de progênies com resistência à mistura populacional de M. incognita raça 1 e M. javanica em campo, Charchar \& Vieira (1994) verificaram diferenças entre as porcentagens de infecção com evidências de existir variação genotípica entre progênies, $o$ que facilita e viabiliza o processo de seleção de cenoura com resistência ao nematóide-das-galhas (Meloidogyne spp.).

No experimento em casa-de-vegetação, houve interações significativas entre espécies/mistura de espécies e os caracteres avaliados. Desta forma os tratamentos foram submetidos ao agrupamento de médias para cada espécie e para a mistura de espécies. Com exceção do FR em campo para ambas as populações e porcentagem de infecção (\% INF) para a população ' 0812518 ', o teste de Scott-Knott a 5\% de probabilidade, possibilitou o agrupamento das progênies e testemunhas de acordo com cada característica avaliada (Tabelas 1 e 2).

Para o caráter rendimento de raiz $(\mathrm{t} /$ ha) em campo, a cultivar Kuronan (testemunha suscetível ao Meloidogyne spp.) obteve os menores valores. $\mathrm{O}$ rendimento de muitas famílias para as duas populações foi superior ao das testemunhas com indicação que estas populações possuem bom potencial produtivo.

A porcentagem de infecção em campo foi significativa apenas para a população ‘0812519’, e não foi possível verificar diferença entre as duas testemunhas. Algumas progênies tiveram menor porcentagem de infecção, porém, pelo valor da média das progênies, foi observado que os três ciclos de seleção 
Tabela 1. Reação de populações de cenoura em campo e em casa-de-vegetação aos nematóides-das-galhas ( $M$. incognita raça 1 , $M$. javanica e mistura populacional de ambos) para progênies da população '0812518' e testemunhas (reaction of carrot populations in field and greenhouse to root-knot nematode (M. incognita race 1, M. javanica and population mix of both) for the progeny of the population '0812518 'and control cultivars). Brasília, Embrapa Hortaliças, 2009.

\begin{tabular}{|c|c|c|c|c|c|c|c|c|c|}
\hline \multirow{2}{*}{ Progênies } & \multicolumn{3}{|c|}{ Campo } & \multicolumn{6}{|c|}{ Casa-de-vegetação } \\
\hline & t/ha & \%INF & FR & IG Inc. & IMO Inc. & IG Jav. & IMO Jav. & IG Mis. & IMO Mis. \\
\hline 01 & $21,63 c$ & $24,40 \mathrm{a}$ & $7,52 \mathrm{a}$ & $4,00 \mathrm{~b}$ & $3,75 \mathrm{~b}$ & $4,00 \mathrm{~b}$ & $3,50 \mathrm{c}$ & $3,25 \mathrm{c}$ & $2,75 \mathrm{c}$ \\
\hline 02 & $20,95 \mathrm{c}$ & $27,79 \mathrm{a}$ & $5,48 \mathrm{a}$ & $4,50 \mathrm{a}$ & $2,25 b$ & $2,50 \mathrm{~b}$ & $1,00 \mathrm{c}$ & $3,00 \mathrm{c}$ & $1,75 \mathrm{c}$ \\
\hline 03 & $15,60 \mathrm{~d}$ & $26,92 \mathrm{a}$ & $5,25 \mathrm{a}$ & $4,50 \mathrm{a}$ & $3,50 \mathrm{a}$ & $3,50 \mathrm{a}$ & $1,75 b$ & $2,50 \mathrm{~d}$ & $1,75 \mathrm{c}$ \\
\hline 04 & $17,70 \mathrm{~d}$ & $32,72 \mathrm{a}$ & $9,18 \mathrm{a}$ & $2,00 \mathrm{e}$ & $1,25 \mathrm{~b}$ & $3,75 \mathrm{a}$ & $1,00 \mathrm{c}$ & $2,00 \mathrm{~d}$ & $1,00 \mathrm{~d}$ \\
\hline 05 & $13,93 \mathrm{~d}$ & $27,21 \mathrm{a}$ & $8,12 \mathrm{a}$ & $3,75 c$ & $2,50 \mathrm{~b}$ & $3,25 \mathrm{a}$ & $2,00 \mathrm{~b}$ & $2,50 \mathrm{~d}$ & $2,00 \mathrm{c}$ \\
\hline 06 & $23,88 \mathrm{c}$ & $30,31 \mathrm{a}$ & $5,93 a$ & $4,65 \mathrm{a}$ & $2,00 \mathrm{~b}$ & $3,25 \mathrm{a}$ & $1,75 b$ & $3,75 b$ & $2,00 \mathrm{c}$ \\
\hline 07 & $16,10 \mathrm{~d}$ & $26,22 a$ & $11,59 \mathrm{a}$ & $3,75 \mathrm{c}$ & $2,25 b$ & $2,50 \mathrm{~b}$ & $1,25 \mathrm{c}$ & $2,50 \mathrm{~d}$ & $1,75 \mathrm{c}$ \\
\hline 08 & $28,80 \mathrm{~b}$ & $20,79 a$ & $5,87 \mathrm{a}$ & $4,75 \mathrm{a}$ & $3,25 \mathrm{a}$ & $3,25 \mathrm{a}$ & $1,50 \mathrm{c}$ & $3,00 \mathrm{c}$ & $1,50 \mathrm{~d}$ \\
\hline 09 & $21,03 \mathrm{c}$ & $25,05 \mathrm{a}$ & $9,60 \mathrm{a}$ & $4,75 \mathrm{a}$ & $4,00 \mathrm{a}$ & $2,50 \mathrm{~b}$ & $1,50 \mathrm{c}$ & $3,75 b$ & $2,25 \mathrm{c}$ \\
\hline 10 & $26,38 \mathrm{c}$ & $20,81 \mathrm{a}$ & $5,99 \mathrm{a}$ & $4,50 \mathrm{a}$ & $2,75 \mathrm{a}$ & $2,75 b$ & $1,00 \mathrm{c}$ & $4,00 \mathrm{~b}$ & $2,00 \mathrm{c}$ \\
\hline 11 & $17,53 \mathrm{~d}$ & $31,83 a$ & $6,89 a$ & $4,33 b$ & $2,33 b$ & $2,75 b$ & $1,00 \mathrm{c}$ & $3,75 b$ & $2,00 \mathrm{c}$ \\
\hline 12 & $30,60 \mathrm{~b}$ & $19,25 \mathrm{a}$ & $8,46 a$ & $3,00 \mathrm{~d}$ & $1,65 b$ & $2,00 \mathrm{~b}$ & $1,00 \mathrm{c}$ & $4,25 \mathrm{a}$ & $1,25 \mathrm{~d}$ \\
\hline 13 & $22,50 \mathrm{c}$ & $15,69 \mathrm{a}$ & $4,59 \mathrm{a}$ & $3,50 \mathrm{c}$ & $2,25 \mathrm{~b}$ & $3,00 \mathrm{a}$ & $2,00 \mathrm{~b}$ & $4,25 \mathrm{a}$ & $4,00 \mathrm{a}$ \\
\hline 14 & $25,18 \mathrm{c}$ & $21,79 \mathrm{a}$ & $11,81 \mathrm{a}$ & $4,00 \mathrm{~b}$ & $3,25 \mathrm{a}$ & $2,25 \mathrm{~b}$ & $1,50 \mathrm{c}$ & $4,50 \mathrm{a}$ & $3,25 \mathrm{a}$ \\
\hline 15 & $14,53 \mathrm{~d}$ & $52,78 \mathrm{a}$ & $8,92 \mathrm{a}$ & $5,00 \mathrm{a}$ & $3,50 \mathrm{a}$ & $2,25 b$ & $1,25 \mathrm{c}$ & $5,00 \mathrm{a}$ & $2,32 \mathrm{c}$ \\
\hline 16 & $25,53 \mathrm{c}$ & $25,08 \mathrm{a}$ & $4,44 \mathrm{a}$ & $5,00 \mathrm{a}$ & $4,00 \mathrm{a}$ & $2,25 \mathrm{~b}$ & $1,00 \mathrm{c}$ & $2,50 \mathrm{~d}$ & $1,75 \mathrm{c}$ \\
\hline 17 & $14,75 d$ & $33,63 a$ & $7,97 \mathrm{a}$ & $3,75 c$ & $3,00 \mathrm{a}$ & $2,50 \mathrm{~b}$ & $1,25 \mathrm{c}$ & $4,00 \mathrm{~b}$ & $2,25 \mathrm{c}$ \\
\hline 18 & $16,95 d$ & $22,44 a$ & $11,08 \mathrm{a}$ & $4,25 b$ & $3,50 \mathrm{a}$ & $1,00 \mathrm{c}$ & $1,00 \mathrm{c}$ & $4,50 \mathrm{a}$ & $2,00 \mathrm{c}$ \\
\hline 19 & $18,20 \mathrm{~d}$ & $27,51 \mathrm{a}$ & $7,12 \mathrm{a}$ & $3,65 \mathrm{c}$ & $2,33 b$ & $3,33 \mathrm{a}$ & $2,00 \mathrm{~b}$ & $3,75 b$ & $3,00 \mathrm{~b}$ \\
\hline 20 & $42,13 a$ & $29,57 a$ & $4,26 \mathrm{a}$ & $4,65 \mathrm{a}$ & $3,33 \mathrm{a}$ & $2,65 b$ & $1,33 \mathrm{c}$ & $4,75 \mathrm{a}$ & $3,00 \mathrm{~b}$ \\
\hline 21 & $25,53 \mathrm{c}$ & $28,09 \mathrm{a}$ & $7,14 \mathrm{a}$ & $4,00 \mathrm{~b}$ & $3,75 \mathrm{a}$ & $2,75 b$ & $1,25 \mathrm{c}$ & $4,50 \mathrm{a}$ & $2,00 \mathrm{c}$ \\
\hline 22 & $15,00 \mathrm{~d}$ & $24,80 \mathrm{a}$ & $5,86 \mathrm{a}$ & $3,75 c$ & $2,25 b$ & $2,50 \mathrm{~b}$ & $1,50 \mathrm{c}$ & $2,50 \mathrm{~d}$ & $2,00 \mathrm{c}$ \\
\hline 23 & $13,88 \mathrm{~d}$ & $23,64 a$ & $4,73 \mathrm{a}$ & $4,00 \mathrm{~b}$ & $3,25 \mathrm{a}$ & $3,50 \mathrm{a}$ & $2,50 \mathrm{a}$ & $4,25 \mathrm{a}$ & $2,00 \mathrm{c}$ \\
\hline 24 & $21,53 \mathrm{c}$ & $21,54 \mathrm{a}$ & $4,52 \mathrm{a}$ & $4,75 \mathrm{a}$ & $3,50 \mathrm{a}$ & $2,75 b$ & $1,75 \mathrm{~b}$ & $3,50 \mathrm{~b}$ & $2,25 \mathrm{c}$ \\
\hline 25 & $21,70 \mathrm{c}$ & $36,29 a$ & $4,97 \mathrm{a}$ & $4,25 \mathrm{~b}$ & $4,00 \mathrm{a}$ & $2,25 b$ & $1,00 \mathrm{c}$ & $2,75 d$ & $1,75 \mathrm{c}$ \\
\hline 26 & $28,73 b$ & $42,87 \mathrm{a}$ & $1,83 \mathrm{a}$ & $4,50 \mathrm{a}$ & $3,25 \mathrm{a}$ & $3,00 \mathrm{a}$ & $1,00 \mathrm{c}$ & $4,00 \mathrm{~b}$ & $2,00 \mathrm{c}$ \\
\hline 27 & $20,10 \mathrm{~d}$ & $35,43 a$ & $5,04 \mathrm{a}$ & $3,75 c$ & $3,00 \mathrm{a}$ & $3,75 a$ & $2,00 \mathrm{~b}$ & $4,25 \mathrm{a}$ & $2,00 \mathrm{c}$ \\
\hline 28 & $32,13 b$ & $27,71 \mathrm{a}$ & $7,04 a$ & $3,75 \mathrm{c}$ & $2,50 \mathrm{~b}$ & $2,65 b$ & $1,33 \mathrm{c}$ & $4,25 \mathrm{a}$ & $2,00 \mathrm{c}$ \\
\hline 29 & $24,75 \mathrm{c}$ & $33,00 \mathrm{a}$ & $3,92 \mathrm{a}$ & $3,75 \mathrm{c}$ & $2,00 \mathrm{~b}$ & $3,25 \mathrm{a}$ & $1,50 \mathrm{c}$ & $4,25 \mathrm{a}$ & $3,50 \mathrm{a}$ \\
\hline 30 & $24,30 \mathrm{c}$ & $22,13 a$ & $4,40 \mathrm{a}$ & $3,25 \mathrm{c}$ & $2,00 \mathrm{~b}$ & $2,00 \mathrm{~b}$ & $1,50 \mathrm{c}$ & $4,00 \mathrm{~b}$ & $2,00 \mathrm{c}$ \\
\hline 31 & $29,61 b$ & $20,47 a$ & $12,39 a$ & $2,75 d$ & $1,75 \mathrm{~b}$ & $2,25 \mathrm{~b}$ & $1,00 \mathrm{c}$ & $4,25 \mathrm{a}$ & $2,00 \mathrm{c}$ \\
\hline 32 & $27,55 b$ & $32,94 a$ & $8,09 \mathrm{a}$ & $2,50 \mathrm{~d}$ & $1,75 b$ & $3,50 \mathrm{a}$ & $1,50 \mathrm{c}$ & $3,75 b$ & $2,50 \mathrm{c}$ \\
\hline 33 & $19,53 \mathrm{~d}$ & $35,39 a$ & $5,85 \mathrm{a}$ & $3,50 \mathrm{c}$ & $2,25 \mathrm{~b}$ & $3,00 \mathrm{a}$ & $2,00 \mathrm{~b}$ & $3,65 b$ & $1,32 \mathrm{~d}$ \\
\hline 34 & $27,28 b$ & $30,34 \mathrm{a}$ & $9,13 \mathrm{a}$ & $3,75 \mathrm{c}$ & $2,75 \mathrm{a}$ & $2,75 b$ & $1,25 \mathrm{c}$ & $4,00 \mathrm{~b}$ & $2,00 \mathrm{c}$ \\
\hline 35 & $23,20 \mathrm{c}$ & $22,34 a$ & $9,36 \mathrm{a}$ & $3,50 \mathrm{c}$ & $2,25 b$ & $2,00 \mathrm{~b}$ & $1,25 \mathrm{c}$ & $3,50 \mathrm{~b}$ & $2,00 \mathrm{c}$ \\
\hline 36 & $27,23 b$ & $38,33 a$ & $3,68 \mathrm{a}$ & $5,00 \mathrm{a}$ & $3,00 \mathrm{a}$ & $2,50 \mathrm{~b}$ & $1,25 \mathrm{c}$ & $4,00 \mathrm{~b}$ & $2,75 b$ \\
\hline 37 & $29,68 b$ & $45,11 \mathrm{a}$ & $5,38 \mathrm{a}$ & $5,00 \mathrm{a}$ & $3,25 \mathrm{a}$ & $3,75 a$ & $2,00 \mathrm{~b}$ & $3,75 b$ & $2,00 \mathrm{c}$ \\
\hline 38 & $23,33 \mathrm{c}$ & $33,12 \mathrm{a}$ & $5,29 a$ & $3,75 \mathrm{c}$ & $2,00 \mathrm{~b}$ & $3,25 \mathrm{a}$ & $2,00 \mathrm{~b}$ & $4,65 \mathrm{a}$ & $2,00 \mathrm{c}$ \\
\hline Brasília & $11,79 \mathrm{~d}$ & $28,72 \mathrm{a}$ & $4,18 \mathrm{a}$ & $4,00 \mathrm{~b}$ & $2,25 \mathrm{~b}$ & $2,25 \mathrm{~b}$ & $1,50 \mathrm{c}$ & $3,25 \mathrm{c}$ & $2,00 \mathrm{~b}$ \\
\hline Kuronan & $4,29 \mathrm{e}$ & $48,14 \mathrm{a}$ & $6,09 a$ & $4,00 \mathrm{~b}$ & $3,75 a$ & $4,00 \mathrm{a}$ & $3,50 \mathrm{a}$ & $3,25 \mathrm{c}$ & $3,50 \mathrm{a}$ \\
\hline Média & 22,86 & 28,82 & 6,81 & 3,99 & 2,76 & 2,80 & 1,50 & 3,71 & 2,14 \\
\hline$h^{2}(\%)$ & 0,88 & 0,52 & 0,35 & 0,84 & 0,85 & 0,78 & 0,69 & 0,86 & 0,84 \\
\hline CV (\%) & 11,11 & 21,11 & 28,98 & 6,44 & 9,05 & 8,95 & 13,12 & 6,80 & 9,15 \\
\hline
\end{tabular}

\%INF= porcentagem de infecção; $\mathrm{FR}=$ fator de reprodução; $\mathrm{IG}=$ índice de galhas; $\mathrm{IMO}=$ índice de massa de ovos; Inc.= Meloidogyne incognita raça 1; Jav.= Meloidogyne javanica; Mis.= mistura de Meloidogyne incognita raça 1 e Meloidogyne javanica $; \mathrm{h}^{2}=$ herdabilidade no sentido amplo; Médias seguidas da mesma letra na coluna não diferem entre si pelo teste de Scott-Knott a 5\% de probabilidade $(\% \mathrm{INF}=$ percentage of infection; $\mathrm{FR}=$ reproduction factor; $\mathrm{IG}=$ gall index; $\mathrm{IMO}=$ mass index of eggs; Inc.= Meloidogyne incognita race 1 , Jav.= Meloidogyne javanica; Mis.= mixture of Meloidogyne incognita race 1 and Meloidogyne javanica; $\mathrm{h}^{2}=$ broad-sense heritability; Means followed by the same letter in column do not differ by Scott-Knott at $5 \%$ probability). 
Tabela 2. Reação de populações de cenoura em campo e em casa-de-vegetação, aos nematóides-das-galhas (M. incognita raça 1, M. javanica e mistura populacional de ambos) para progênies da população '0812519' e cultivares testemunhas (reaction of carrot populations in field and greenhouse, to root-knot nematode (M. incognita race 1, M. javanica and population mix of both) for the progeny of the population '0812519 'and control cultivars). Brasília, Embrapa Hortaliças, 2009.

\begin{tabular}{|c|c|c|c|c|c|c|c|c|c|}
\hline \multirow{2}{*}{ Progênies } & \multicolumn{3}{|c|}{ Campo } & \multicolumn{6}{|c|}{ Casa-de-vegetação } \\
\hline & t/ha & $\%$ INF & FR & IG Inc. & IMO Inc. & IG Jav. & IMO Jav. & IG Mis. & IMO Mis. \\
\hline 01 & $27,80 \mathrm{a}$ & $34,98 \mathrm{a}$ & $5,94 \mathrm{a}$ & $4,25 \mathrm{a}$ & $4,00 \mathrm{a}$ & $2,50 \mathrm{~d}$ & $1,75 b$ & $2,50 \mathrm{~b}$ & $2,75 \mathrm{a}$ \\
\hline 02 & $16,00 \mathrm{c}$ & $24,98 b$ & $6,17 \mathrm{a}$ & $3,50 \mathrm{~b}$ & $2,00 \mathrm{~b}$ & $2,25 \mathrm{~d}$ & $1,25 \mathrm{c}$ & $3,00 \mathrm{~b}$ & $1,75 b$ \\
\hline 03 & $25,30 \mathrm{~b}$ & $17,80 \mathrm{~b}$ & $7,13 \mathrm{a}$ & $4,33 \mathrm{a}$ & $2,65 b$ & $2,00 \mathrm{~d}$ & $1,50 \mathrm{c}$ & $3,75 \mathrm{a}$ & $2,25 \mathrm{a}$ \\
\hline 04 & $22,93 b$ & $29,87 \mathrm{a}$ & $10,27 \mathrm{a}$ & $3,00 \mathrm{~b}$ & $3,00 \mathrm{a}$ & $2,00 \mathrm{~d}$ & $1,00 \mathrm{c}$ & $3,75 \mathrm{a}$ & $1,50 \mathrm{~b}$ \\
\hline 05 & $37,00 \mathrm{a}$ & $32,32 \mathrm{a}$ & $10,89 \mathrm{a}$ & $4,00 \mathrm{a}$ & $2,33 b$ & $2,25 \mathrm{~d}$ & $1,75 b$ & $2,75 b$ & $2,25 \mathrm{a}$ \\
\hline 06 & $32,08 \mathrm{a}$ & $20,57 b$ & $7,04 \mathrm{a}$ & $4,25 \mathrm{a}$ & $3,25 \mathrm{a}$ & $2,00 \mathrm{~d}$ & $2,00 \mathrm{~b}$ & $3,25 \mathrm{a}$ & $2,00 \mathrm{~b}$ \\
\hline 07 & $14,53 \mathrm{c}$ & $23,82 b$ & $5,03 \mathrm{a}$ & $4,00 \mathrm{a}$ & $2,33 b$ & $2,50 \mathrm{~d}$ & $1,50 \mathrm{c}$ & $2,50 \mathrm{~b}$ & $1,25 b$ \\
\hline 08 & $13,88 \mathrm{c}$ & $38,23 \mathrm{a}$ & $5,55 \mathrm{a}$ & $4,25 \mathrm{a}$ & $2,50 \mathrm{~b}$ & $2,00 \mathrm{~d}$ & $1,75 b$ & $3,25 \mathrm{a}$ & $2,25 \mathrm{a}$ \\
\hline 09 & $28,83 \mathrm{a}$ & $17,15 b$ & $10,64 a$ & $4,50 \mathrm{a}$ & $2,50 \mathrm{~b}$ & $2,25 \mathrm{~d}$ & $1,00 \mathrm{c}$ & $2,65 b$ & $1,65 b$ \\
\hline 10 & $15,63 \mathrm{c}$ & $32,72 \mathrm{a}$ & 4,28a & $4,00 \mathrm{a}$ & $2,75 b$ & $2,00 \mathrm{~d}$ & $1,50 \mathrm{c}$ & $4,50 \mathrm{a}$ & $3,00 \mathrm{a}$ \\
\hline 11 & $23,28 b$ & $31,54 \mathrm{a}$ & $5,03 \mathrm{a}$ & $4,00 \mathrm{a}$ & $3,50 \mathrm{a}$ & $2,00 \mathrm{~d}$ & $1,25 \mathrm{c}$ & $3,50 \mathrm{a}$ & $2,00 \mathrm{~b}$ \\
\hline 12 & $21,93 b$ & $31,54 \mathrm{a}$ & $6,31 \mathrm{a}$ & $4,33 a$ & $2,65 b$ & $2,75 c$ & $3,00 \mathrm{a}$ & $3,00 \mathrm{~b}$ & $2,50 \mathrm{a}$ \\
\hline 13 & $18,50 \mathrm{c}$ & $16,16 b$ & $4,93 a$ & $4,25 \mathrm{a}$ & $3,25 \mathrm{a}$ & $2,00 \mathrm{~d}$ & $1,00 \mathrm{c}$ & $2,75 b$ & $1,50 \mathrm{~b}$ \\
\hline 14 & $27,15 \mathrm{a}$ & $19,00 \mathrm{~b}$ & $4,59 a$ & $3,65 b$ & $2,65 b$ & $2,00 \mathrm{~d}$ & $1,25 \mathrm{c}$ & $4,25 \mathrm{a}$ & $3,25 \mathrm{a}$ \\
\hline 15 & $26,05 b$ & $19,09 b$ & $7,06 \mathrm{a}$ & $3,25 b$ & $2,25 b$ & $3,25 b$ & $1,75 b$ & $3,00 \mathrm{~b}$ & $1,25 b$ \\
\hline 16 & $11,28 \mathrm{c}$ & $27,78 b$ & $6,68 \mathrm{a}$ & $4,75 a$ & $2,00 \mathrm{~b}$ & $2,25 \mathrm{~d}$ & $1,75 b$ & $3,00 \mathrm{~b}$ & $2,25 \mathrm{a}$ \\
\hline 17 & $14,98 \mathrm{c}$ & $37,71 \mathrm{a}$ & $20,39 a$ & $3,75 b$ & $2,00 \mathrm{~b}$ & $2,00 \mathrm{~d}$ & $1,50 \mathrm{c}$ & $4,00 \mathrm{a}$ & $3,00 \mathrm{a}$ \\
\hline 18 & $37,30 \mathrm{a}$ & $12,45 b$ & 6,06a & $4,00 \mathrm{a}$ & $2,00 \mathrm{~b}$ & $2,00 \mathrm{~d}$ & $1,75 b$ & $4,25 \mathrm{a}$ & $2,75 a$ \\
\hline 19 & $29,30 \mathrm{a}$ & $26,67 b$ & $3,35 \mathrm{a}$ & $3,75 b$ & $2,00 \mathrm{~b}$ & $2,25 d$ & $1,00 \mathrm{c}$ & $3,50 \mathrm{a}$ & $3,00 \mathrm{a}$ \\
\hline 20 & $20,00 c$ & $34,56 a$ & $11,19 \mathrm{a}$ & $4,50 \mathrm{a}$ & $2,75 b$ & $2,00 \mathrm{~d}$ & $1,00 \mathrm{c}$ & $4,50 \mathrm{a}$ & $3,00 \mathrm{a}$ \\
\hline 21 & $29,75 a$ & $24,06 \mathrm{~b}$ & $10,81 \mathrm{a}$ & $4,25 \mathrm{a}$ & $2,00 \mathrm{~b}$ & $2,25 \mathrm{~d}$ & $1,75 b$ & $2,50 \mathrm{~b}$ & $1,50 \mathrm{~b}$ \\
\hline 22 & $30,43 a$ & $23,46 b$ & 6,90a & $3,00 \mathrm{~b}$ & $2,00 \mathrm{~b}$ & $2,00 \mathrm{~d}$ & $1,00 \mathrm{c}$ & $2,00 \mathrm{~b}$ & $1,75 b$ \\
\hline 23 & $27,10 \mathrm{a}$ & $29,12 \mathrm{a}$ & $8,05 \mathrm{a}$ & $4,50 \mathrm{a}$ & $4,25 \mathrm{a}$ & $3,00 \mathrm{c}$ & $2,00 \mathrm{~b}$ & $3,75 \mathrm{a}$ & $3,25 \mathrm{a}$ \\
\hline 24 & $12,70 \mathrm{c}$ & $34,09 a$ & $8,54 a$ & $4,75 \mathrm{a}$ & $3,50 \mathrm{a}$ & $2,25 \mathrm{~d}$ & $1,75 b$ & $3,00 \mathrm{~b}$ & $1,50 \mathrm{~b}$ \\
\hline 25 & $29,48 \mathrm{a}$ & $25,63 b$ & $10,63 \mathrm{a}$ & $3,50 \mathrm{~b}$ & $2,00 \mathrm{~b}$ & $2,00 \mathrm{~d}$ & $2,00 \mathrm{~b}$ & $3,75 a$ & $2,25 \mathrm{a}$ \\
\hline 26 & $28,90 \mathrm{a}$ & $26,79 a$ & $8,33 \mathrm{a}$ & $3,25 b$ & $2,25 b$ & $2,75 \mathrm{c}$ & $1,75 b$ & $2,00 \mathrm{~b}$ & $1,00 \mathrm{~b}$ \\
\hline 27 & $20,58 b$ & $26,65 b$ & $6,91 \mathrm{a}$ & $4,25 \mathrm{a}$ & $3,75 a$ & $2,75 \mathrm{c}$ & $1,75 b$ & $3,33 a$ & $2,33 a$ \\
\hline 28 & $27,05 \mathrm{a}$ & $22,08 b$ & $8,84 a$ & $4,00 \mathrm{a}$ & $3,25 \mathrm{a}$ & $3,25 b$ & $1,50 \mathrm{c}$ & $3,50 \mathrm{a}$ & $2,75 \mathrm{a}$ \\
\hline 29 & $20,60 c$ & $24,03 b$ & $4,13 \mathrm{a}$ & $4,00 \mathrm{a}$ & $3,25 \mathrm{a}$ & $2,00 \mathrm{~d}$ & $1,25 \mathrm{c}$ & $3,33 \mathrm{a}$ & $2,33 a$ \\
\hline 30 & $16,35 \mathrm{c}$ & $17,10 \mathrm{~b}$ & $11,16 \mathrm{a}$ & $3,00 \mathrm{~b}$ & $2,75 b$ & $2,75 \mathrm{c}$ & $1,75 b$ & $2,00 \mathrm{~b}$ & $2,00 \mathrm{~b}$ \\
\hline 31 & $17,23 \mathrm{c}$ & $40,10 \mathrm{a}$ & $10,96 a$ & $3,75 b$ & $2,75 b$ & $2,50 \mathrm{~d}$ & $2,25 b$ & $2,75 b$ & $1,75 b$ \\
\hline Brasília & $11,79 \mathrm{c}$ & $28,72 \mathrm{a}$ & 4,18a & $4,00 \mathrm{a}$ & $2,25 b$ & $2,25 \mathrm{~d}$ & $1,50 \mathrm{c}$ & $3,25 \mathrm{a}$ & $2,00 \mathrm{~b}$ \\
\hline Kuronan & 4,29d & $48,14 \mathrm{a}$ & $6,09 a$ & $4,00 \mathrm{a}$ & $3,75 \mathrm{a}$ & $4,00 \mathrm{a}$ & $3,50 \mathrm{a}$ & $3,25 \mathrm{a}$ & $3,50 \mathrm{a}$ \\
\hline Média & 23,35 & 26,51 & 7,86 & 3,95 & 2,71 & 2,31 & 1,58 & 3,21 & 2,17 \\
\hline$h^{2}(\%)$ & 0,89 & 0,52 & 0,42 & 0,58 & 0,78 & 0,78 & 0,65 & 0,76 & 0,79 \\
\hline CV (\%) & 12,12 & 20,62 & 29,98 & 7,02 & 9,31 & 7,23 & 13,83 & 8,90 & 11,47 \\
\hline
\end{tabular}

\%INF= porcentagem de infecção; $\mathrm{FR}=$ fator de reprodução; $\mathrm{IG}=$ índice de galhas; $\mathrm{IMO}=$ índice de massa de ovos; Inc.= Meloidogyne incognita raça 1; Jav.= Meloidogyne javanica; Mis.= mistura de Meloidogyne incognita raça 1 e Meloidogyne javanica; $\mathrm{h}^{2}=$ herdabilidade no sentido amplo; Médias seguidas da mesma letra na coluna não diferem entre si pelo teste de Scott-Knott a 5\% de probabilidade $(\% \mathrm{INF}=$ percentage of infection; $\mathrm{FR}=$ reproduction factor; $\mathrm{IG}=$ gall index; $\mathrm{IMO}=$ mass index of eggs; Inc.= Meloidogyne incognita race 1, Jav.= Meloidogyne javanica; Mis.= mixture of Meloidogyne incognita race 1 and Meloidogyne javanica; $\mathrm{h}^{2}=$ broad-sense heritability; Means followed by the same letter in column do not differ by Scott-Knott at $5 \%$ probability). 
Tabela 3. Correlação fenotípica entre caracteres relacionados aos nematóides-das-galhas em cenoura avaliados em campo e em casa de vegetação para a população '0812518' (diagonal inferior) e '0812519' (diagonal superior) (phenotypic correlation among traits related to root-knot nematode in carrots evaluated in field and greenhouse for the population '0812518 '(lower diagonal) and '0812519' (upper diagonal). Brasília, Embrapa Hortaliças, 2009.

\begin{tabular}{lcccccccc}
\hline & t/ha & \%INF & IG Inc & IMO Inc & IG Jav & IMO Jav & IG Mis & IMO Mis \\
\hline t/ha & 1 & $-0,71^{*}$ & $-0,17$ & $-0,18$ & $-0,34^{*}$ & $-0,36^{*}$ & 0,01 & $-0,10$ \\
\%INF & $-0,42^{*}$ & 1 & $0,33^{*}$ & $0,48^{*}$ & $0,32^{*}$ & $0,68^{*}$ & 0,15 & $0,39^{*}$ \\
IG Inc & 0,03 & $0,22^{*}$ & 1 & $0,27^{*}$ & $-0,17$ & 0,15 & $0,33^{*}$ & $0,35^{*}$ \\
IMO Inc & $-0,12$ & $0,32^{*}$ & $0,80^{*}$ & 1 & $0,43^{*}$ & $0,30^{*}$ & 0,07 & 0,18 \\
IG Jav & $-0,19$ & $0,67^{*}$ & $-0,13$ & $-0,10$ & 1 & $0,61 *$ & $-0,26^{*}$ & 0,02 \\
IMO Jav & $-0,62^{*}$ & $0,60^{*}$ & 0,00 & 0,16 & $0,63^{*}$ & 1 & $-0,14$ & 0,17 \\
IG Mis & $0,45^{*}$ & $-0,01$ & 0,10 & 0,09 & $-0,15$ & 0,05 & 1 & $0,69^{*}$ \\
IMO Mis & $-0,08$ & 0,04 & 0,19 & 0,13 & 0,01 & $0,42^{*}$ & $0,40^{*}$ & 1 \\
\hline
\end{tabular}

\%INF.= porcentagem de infecção; $\mathrm{FR}=$ fator de reprodução; $\mathrm{IG}=$ índice de galhas; $\mathrm{IMO}=$ índice de massa de ovos; Inc.= Meloidogyne incognita raça 1; Jav.= M. javanica; Mis.= mistura de $M$. incognita raça 1 e $M$. javanica. *Significativo a $5 \%$ pelo teste de $t(\% \mathrm{INF} .=$ percentage of infection; $\mathrm{FR}=$ reproduction factor; $\mathrm{IG}=$ gall index; $\mathrm{IMO}=$ index of eggs mass; Inc.= Meloidogyne incognita race $1 ; \mathrm{Jav}$.= M. javanica; Mis. $=$ mixture of $M$. incognita race 1 and $M$. javanica. ${ }^{*}$ Significant at $5 \%$ by $t$ test $)$.

aplicados em campo não possibilitaram ganhos consideráveis em relação à cultivar 'BRS Brasília', que originou estas populações. Isso foi confirmado pelos testes em casa-de-vegetação, indicando que a seleção empregada nestas populações não foi efetiva para aumentar o nível de tolerância aos nematóides, com necessidades de ajustes na metodologia de seleção de cenoura para resistência ao nematóide-das-galhas. No entanto, algumas progênies tiveram menor porcentagem de infecção do que a testemunha tolerante 'BRS Brasília'. Isto demonstra que após o aprimoramento da metodologia de experimentação, poderá haver uma potencial variabilidade para seleção com o objetivo de aumentar o nível de tolerância ao nematóide-das-galhas nestas duas populações. A possibilidade de obtenção de ganhos para a resistência às duas espécies de nematóides avaliadas, para populações do grupo Brasília, também foi observada por Vieira et al. (2003).

Para os caracteres avaliados em casa-de-vegetação, a testemunha 'Kuronan' foi um dos materiais mais suscetíveis e 'BRS Brasília' um dos mais tolerantes aos nematóides, conforme esperado. No entanto, para a variável índice de galhas (IG) para M. incognita e para a mistura populacional de $M$. incognita raça 1 e $M$. javanica, as testemunhas não diferiram entre si. Indicando que a verificação do IG não foi eficiente em discriminar a diferença no nível de resistência que existe entre estas cultivares.
Pelos valores das médias, pode-se verificar que as duas populações foram mais tolerantes à M. javanica. Para esta espécie, em casa-de-vegetação, o índice de galhas e o índice de massa de ovos foram eficientes na discriminação das testemunhas suscetíveis e resistentes. Para $M$. incognita e a mistura de populações de nematóides o índice de massa de ovos foi mais eficiente que o índice de galhas. Devido ao fato de que a testemunha suscetível obteve menores rendimentos que a testemunha tolerante, pode-se inferir que a metodologia mais eficiente para a seleção de genótipos de cenoura resistentes aos nematóides é a avaliação do índice de massa de ovos em casa-de-vegetação associado ao rendimento de raízes em campo. Para $M$. javanica poderia ser acrescentada a avaliação do índice de galhas.

As famílias 01,30 e 35 se destacaram na população '0912518', não estando agrupadas entre as famílias mais suscetíveis para ambas as espécies de nematóides e para a mistura destes, e tiveram rendimentos superiores a 20 t/ha. Para a população '0912519', destaca-se a família 22 , que também não foi agrupada entre as mais suscetíveis e foi agrupada entre as mais produtivas.

De acordo com os valores de correlação fenotípica (Tabela 3), observou-se correlação negativa entre os caracteres rendimento e porcentagem de infecção, ou seja, quanto maior a porcentagem de infecção das raízes, menores foram os rendimentos, o que já era esperado; porém a correlação foi significativa, mas de valor baixo a médio $(-0,42$ e -0,71). Este fato indica que esta variável não consegue explicar completamente o efeito dos nematóides-das-galhas no rendimento de raízes. Da mesma forma, as variáveis IG e IMO, avaliadas em casa-de-vegetação, estiveram correlacionadas dentro de cada espécie de nematóides bem como para a mistura populacional, com evidências de que o aumento de um caráter eleva o outro, o que também seria esperado, porém, as correlações não foram muito fortes: 0,80, 0,63 e 0,40 para $M$. incognita, $M$. javanica e mistura populacional, respectivamente, para a população '0812518'; e, 0,27, 0,61 e 0,69 para M. incognita, $M$. javanica e mistura populacional, respectivamente, para a população '0812519'; indicando que a avaliação de um destes caracteres não substitui o resultado do outro, sendo necessárias ambas as avaliações.

Para a associação entre os caracteres de campo e de casa-de-vegetação, verificou-se que o caráter rendimento foi correlacionado com o caráter IMO para $M$. javanica, ou seja, quanto maior o valor deste índice na casa-de-vegetação, menor foi o rendimento de raízes no campo, mas a correlação foi apenas mediana a baixa $(-0,36$ e $-0,62$ para as populações '0912519' e '0912518', respectivamente). $\mathrm{O}$ rendimento em campo também se correlacionou com o IG para $M$. javanica na população ‘0912519' e para a mistura de populações de nematóides 
para a população ‘0912518'. Já o caráter porcentagem de infecção em campo foi correlacionado positivamente com os caracteres IMO e IG para cada uma das espécies de nematóides, principalmente para $M$. javanica, porém também com magnitudes não muito elevadas. No entanto, o mesmo não ocorreu para a mistura populacional de nematóides.

Na seleção de progênies com resistência à mistura populacional de M. incognita raça 1 e $M$. javanica em campo, Charchar \& Vieira (1994), não observaram correlação entre a produtividade e resistência a nematóides nas progênies selecionadas.

A baixa correlação entre os caracteres medidos dentro de cada ambiente, bem como entre as diferentes condições de seleção, não proporcionou a possibilidade de seleção apenas em um ambiente ou a eliminação de caracteres. Indicando que para a obtenção de cultivares mais produtivas e mais tolerantes aos nematóides-das-galhas é necessária a seleção combinada nos diferentes caracteres e ambientes. A não obtenção de ganhos em relação a cultivar 'BRS Brasília', que originou as populações em avaliação utilizando a metodologia para avaliação em campo, determina a necessidade da busca por métodos mais eficientes de seleção e a necessidade de novas pesquisas nesta área.

\section{REFERÊNCIAS}

BONETTI JIS; FERRAZ S. 1981. Modificações do método de Hussey \& Barker para extração de ovos de Meloidogyne exigua em raízes de cafeeiro. Fitopatologia Brasileira 6: 553.

BRUNO RLA; VIANA JS; SILVA VF; BRUNO GB; MOURA MF. 2007. Produção e qualidade de sementes e raízes de cenoura cultivada em solo com adubação orgânica e mineral. Horticultura Brasileira 25: 170-174.

CARNEIRO RMDG; ALMEIDA MRA. 2001. Técnica de eletroforese usada no estudo de enzimas dos nematóides de galhas para identificação de espécies. Nematologia Brasileira 25: 35-44.

CHARCHAR JM; ARAGÃO FAS. 2003. Sequência de cultivos no controle de Meloidogyne javanica em campo. Nematologia Brasileira 27: 81-86.

CHARCHAR JM; VIEIRA JV. 1994. Seleção de cenoura com resistência a nematóides de galhas (Meloidogyne spp,). Horticultura Brasileira 12: 144-148.

CHARCHAR JM; GONZAGA V; GIORDANO LB; BOITEUX LS; REIS NVB; ARAGÃO FAS. 2003. Reações de cultivares de tomate à infecção por população mista de Meloidogyne incognita raça 1 e $M$. javanica em estufa plástica e campo. Nematologia Brasileira 27: 49-54.

CHARCHAR JM; GONZAGA V; VIEIRA JV; OLIVEIRA VR; MOITA AW. 2007a. Efeito de nematicidas fumigantes e não fumigantes no controle de Meloidogyne spp. em batata e cenoura. Nematologia Brasileira 31: 59-66.

CHARCHAR JM; GONZAGA V; VIEIRA JV; OLIVEIRA VR; MOITA AW; ARAGÃO FAS. 2007b. Efeito da rotação de culturas no controle de Meloidogyne spp. em cenoura na região norte do Estado de Minas Gerais. Nematologia Brasileira 31: 173-179.

CHARCHAR JM; VIEIRA JV; OLIVEIRA VR; MOITA AW. 2009. Cultivo e Incorporação de Leguminosas, Gramíneas e Outras Plantas no controle de Meloidogyne incognita Raça 1 em Cenoura 'Nantes'. Nematologia Brasileira 33: 139-146.

COVOLO G; BENETTI E. 1981. Comportamento de algumas cultivares de cenoura (Daucus carota L,) ao nematóide Meloidogyne javanica (Treub, 1885) Chitwood, 1949. Revista Centro Ciências Rurais 11: 163-168.
CRUZ CD. 2006. Programa Genes - estatística experimental e matrizes. Viçosa: Editora UFV. 285p.

DICKSON DW; STRUBLE FB. 1965. A sievingstaining technique for extraction of egg mass of Meloidogyne incognita from soil. Phytopathology 55: 497.

EISENBACK JD; HIRSCHMANNTRIANTAPHYLLOU H. 1991. Root-knot nematodes: Meloidogyne species and races. In: NICKLE WR (ed). Manual of Agricultural Nematology, New York: Marcel Dekker, Inc, p. 191-274.

HUANG SP; DELLA VECHHIA, PT; FERREIRA PE. 1986a. Varietal response and estimates of heritability of resistance to Meloidogyne javanica in carrots. Journal Nematology 18: 406-501.

HUANG SP; MIRANDA JEC; MALUF WR. 1986b. Resistance to root-knot nematodes in a Brazilian sweet potato collection. Fitopatologia Brasileira 11: 761-767.

JENKINS WR. 1964. A rapid centrifugal flotation technique for separating nematode from soil. Plant Disease Reporter 48: 62.

OOSTENBRINK M. 1966. Major characteristics of the relation between nematodes and plants. Mededelingen Landbouw 66: 1-46.

SCOTT AJ; KNOTT M. 1974. A cluster analysis method for grouping means in the analysis of variance. Biometrics 30: 507-512.

SIMON PW; MATTHEWS W; ROBERTS PA. 2000. Evidence for simply inherited dominant resistance to Meloidogyne javanica in carrot. Theoretical and Applied Genetics 100: 735742.

VIEIRA JV; CHARCHAR JM; ARAGÃO FAS; BOITEUX LS. 2003. Heritability and gain from selection for field resistance against multiple root-knot nematode species (Meloidogyne incognita race 1 and $M$. javanica) in carrot. Euphytica 130: 11-16.

VIEIRA JV; SILVA JBC; CHARCHAR JM; RESENDE FV; FONSECA MEN; CARVALHO AM; MACHADO CMM. 2005. Esplanada: cultivar de cenoura de verão para fins de processamento. Horticultura Brasileira 23: $851-852$. 